\title{
Haben Männer die schlechtere Lobby als Frauen?
}

Heinrich Gretener

Korrespondenz:

Dr. med. Heinrich Gretener

Rigistrasse 1

CH-6330 Cham

hgret[at]bluewin.ch
Das Swiss Medical Board (SMB) kommt in seiner Publikation [1] zum Schluss, dass die Bestimmung des PSA-Wertes im Hinblick auf die Früherkennung eines Prostatakarzinoms bei diesbezüglich symptomlosen Männern ohne Risikofaktoren nicht gerechtfertigt ist. Das SMB empfiehlt sogar, dass die Kosten der PSA-Bestimmung aus obiger Fragestellung nicht mehr zulasten der Krankenversicherung vergütet werden sollen.

Das Medical Board beschäftigt sich nicht nur mit der Analyse der Datenlage zu medizinischen Fragestellungen, sondern fällt auch mit der Aussage auf, es sehe im Zusammenhang mit der PSA-Bestimmung ein Kostenreduktionspotential für das schweizerische Gesundheitswesen von 112 Millionen Franken pro Jahr. Es stützt sich bei dieser Aussage nicht nur auf Daten, sondern macht auch fragwürdige Annahmen. So hat das Medical Board z. B. willkürliche und unva- dem wird in vielen Kantonen der Schweiz das Mamma-Screening bei symptomlosen Frauen über 50 Jahren institutionell durchgeführt (und von den Kostenträgern vergütet). Und dies, obwohl verglichen mit den Studienresultaten zum differenzierten und kritischen Umgang mit der PSA-Bestimmung zur Früherkennung des Prostatakarzinoms der Nutzen des Mamma-Screenings die schlechtere Datenlage aufweist. Vergleichbar verhält es sich mit der Impfung gegen das Humane Papilloma-Virus, die Bestandteil kantonaler Impfprogramme ist und deren Kosten in der ganzen Schweiz von der Grundversicherung übernommen werden.

Seit der weitverbreiteten Einführung des PSATests ist die Sterblichkeit an Prostatakrebs zurückgegangen. Tumoren werden in einem früheren und heilbaren Stadium entdeckt. $\mathrm{Zu}$ einer individuellen Krebsvorsorge des Mannes gehören die PSA-Bestim-

\section{Der PSA-Test ist eine kostengünstige Möglichkeit, Prostatakrebs früh zu erkennen.}

lidierte Annahmen gemacht, welche Lebensqualitätseinbussen die Stresssituationen der PSA-Abklärungen mit sich bringt.

Werden wir also in Zukunft aus Sicht des Swiss Medical Board unser ärztliches Handeln vor allem darauf fokussieren müssen, ob sich unsere Diagnostik und Handlungsweise rein wirtschaftlich rechtfertigt, auch wenn es eine individuelle Realität gibt? Gerade bei Vorsorgeuntersuchungen ist die Number necessary to treat NNT, um ein Leben zu retten beziehungsweise ein Ereignis zu verhindern, hoch. Das ist beim Mamma-Screening so und auch bei der Prävention und dem Screening des Zervixkarzinoms. Und trotz- mung, die klinische Untersuchung und das Abtasten der Prostata. Dies stellt eine kostengünstige Möglichkeit dar, einen Prostatakrebs früh zu erkennen. Wenn nun aufgrund der Analysen und den Empfehlungen des Swiss Medical Board Männern der PSA-Test nicht mehr angeboten werden kann, obwohl die Datenlage den Nutzen dieses Tests belegt, frage ich mich nicht nur als Urologe, sondern auch als Mann, ob wir Männer die schlechtere Lobby haben als die Frauen.

1 Swiss Medical Board PSA-Test nicht geeignet zur Früherkennung des Prostatakarzinoms. Schweiz Ärztezeitung. 2011;92(48):1857. 